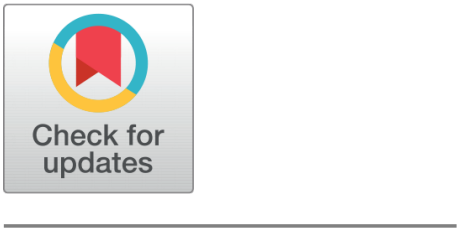

open ACCESS

Received: 24.03.2021

Accepted: 26.04 .2021

Published: 29.05.2021

Citation: Singh SK, Yang L, Hao M, Voulgaris PG (2021) Centralized and Decentralized Schemes for Optimal Scheduling of Electric Vehicles. Indian Journal of Science and Technology 14(19): 1554-1564. https ://doi.org/10.17485/IJST/v14i19.502

* Corresponding author.

santoshkrsingh@intl.zju.edu.cn

Funding: None

Competing Interests: None

Copyright: (c) 2021 Singh et al. This is an open access article distributed under the terms of the Creative Commons Attribution License, which permits unrestricted use, distribution, and reproduction in any medium, provided the original author and source are credited.

Published By Indian Society for Education and Environment (iSee)

ISSN

Print: 0974-6846

Electronic: 0974-5645

\section{Centralized and Decentralized Schemes for Optimal Scheduling of Electric Vehicles}

\author{
Santosh Kumar Singh ${ }^{1}$, Liangjing Yang ${ }^{1}$, Ma Hao ${ }^{1}$, Petros G Voulgaris ${ }^{2}$ \\ 1 Electrical Engineering Department, Zhejiang University-University of Illinois at Urbana \\ Champaign Institute (ZJU-UIUC), Haining, China \\ 2 Mechanical Engineering Department, University of Nevada, Reno, USA
}

\section{Abstract}

Objective: To evaluate the performance of centralized scheduling scheme for charging/discharging of Electric Vehicles (EVs). Methods: To achieve optimal scheduling of EV charging and discharging, two schemes such as centralized and decentralized scheduling schemes are proposed and evaluated in this paper. Both schemes are intended to reshape the load profile through scheduled charging/discharging. Proposed schemes are tested for one day scheduling of 200 EVs. Findings: Optimal scheduling schemes require perfect information of EV and load so that the actual load can be used in simulation. However, actual load in the future interval is impossible to determine. In decentralized scheduling scheme, forecasted loads are used in the simulation. Hence, this scheme is called practical solution of EV scheduling. Novelty: Results demonstrate that, though the centralized scheduling scheme achieved the best results, it is impractical due to its dependency on future data and decentralized scheduling scheme is based on forecasted data which is the practical solution.

Keywords: Electric Vehicle; Vehicle to Grid; Grid to Vehicle

\section{Nomenclature}

$L$ Interval set, $l$ Length of each interval, $E$ Electric vehicle fleet, $n$ Interval number, $Y_{\text {en }}$ Charging/Discharging power of EV in $n$ interval, $P^{n}$ Past interval set of interval n, $A_{e}^{p-i n} \mathrm{EV}$ plug in time, $A_{e}^{p-o u t} \mathrm{EV}$ plug-out time, $A_{e}$ Set of total charging/discharging intervals of EV set, $B_{e}^{i n i}$ Battery initial energy, $B_{e}^{f i n}$ Battery final energy, $B_{e}^{c a p}$ Battery capacity, $R_{e}$ Energy ratio, $C^{\max }$ Maximum charging power, $L_{n}^{a}$ Actual load at $\mathrm{n}$ interval, $L_{o}^{f}$ Forecasted load at interval zero, $D_{i}^{(n)}$ Current ongoing EV set, $S_{i}^{(n)}$ Current sliding window, $S_{e}^{D}$ Starting time of EV charging period, $F_{e}^{D}$ Finishing time of EV charging period, $S_{e}^{S}$ Starting time of sliding window, $F_{e}^{S}$ Finishing time of sliding window

\section{Introduction}

The discharge of toxic and harmful gases like $\mathrm{N}_{2} \mathrm{O}, \mathrm{CH}_{4}$ and $\mathrm{CO}_{2}$ traps the heat of the sun which is radiated back to space from earth, resulting in global warming that leads to melting of snow caps, expanding ocean area and increase in sea level. The 
Inter Governmental Panel (IPC) on climate change forecasts that the rise of temperature from 2.5 to 10 degree Fahrenheit over the next century causes serious problems to human life ${ }^{(1)}$. Transportation, electricity production and industries are main sources of greenhouse gases. Pollution through transportation comes from cars, trucks, ships, planes, trains etc which uses mostly petrol, diesel, and gasoline as fuel. Transportation is one of the main sources of carbon emission. Carbon dioxide levels are one of the main reasons for global climate change. The current level of atmospheric carbon dioxide is 408 parts per million ${ }^{(1)}$.

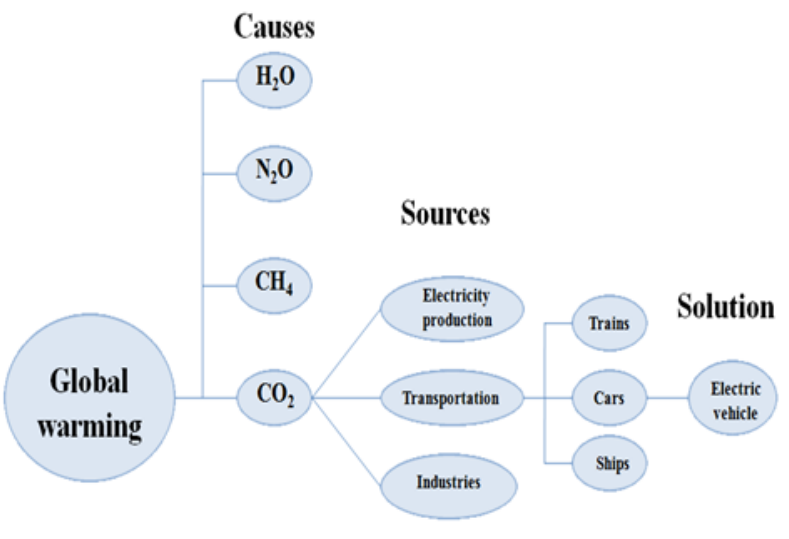

Fig 1. Illustration of environmental issues with solution

To control the limits of excess pollution, electrification is the most important solution. Electric vehicle uses electricity as a fuel and contribute to clean and green environment with zero carbon emissions. EVs have more superior qualities than a conventional vehicle. EVs can use electricity from public places, charging stations and also from renewable energy sources like sun and wind. Along with green environment, EVs provide ancillary services to the grid ${ }^{(2)}$. EVs can enhance the penetration of renewable energy source integration with the grid. EVs can also deal with load imbalances by charging their battery from grid whenever there is low load demand and discharging back to the grid whenever there is high load demand. EV charging is referred as $\mathrm{G} 2 \mathrm{~V}$ and discharging is as $\mathrm{V} 2 \mathrm{G}^{(2)}$.

Although a lot of research works are going worldwide to bring the optimal coordination over charging and discharging of EV fleet without disturbing the load profile to minimize the mismatch between load and power generation along with renewable energy penetration but this problem is still challenging when the loads are frequently changing continuously. The continuous and unpredictable change in load profile integrated with grid calls a smart infrastructure for optimal scheduling of EV charging and discharging to deal with the peaks and valleys in the load profile of the electric system. $\operatorname{In}^{(3)}$, authors used peak load management to schedule EV charging and discharging patterns along with price consideration to meet the peak load demand. A guidance algorithm is proposed to minimize the waiting time for EV fleet to plug-in referred to ${ }^{(3)}$. The distributed coordination method is proposed for EV charging and discharging including Var compensation using quadratically constrained programming formulation. Proposed scheme improves the speed and computational time for EV scheduling with respect to load demand and Var compensation ${ }^{(4)}$.

Three strategies for charging/discharging coordination of EV are proposed in ${ }^{(5)}$ to maximize the parking profits. These strategies include the constraints of EV based on arrival, departure, state of charge and exchange of power between EV fleet in a parking place and the distribution system ${ }^{(5)}$. A decentralized dispatch system is proposed in ${ }^{(6)}$. Optimal coordination problem of renewable energy sources and EVs is solved by $\mathrm{A}^{*}$ search method, to provide effective load leveling ${ }^{(6)}$. Coordination of EV charging and discharging through distributed mechanism is proposed in ${ }^{(7)}$. This concept includes three control algorithms namely day ahead optimal EV scheduling algorithm, hourly distributed EV energy coordination algorithm and short term distributed dispatch algorithm where the first algorithm is for day ahead EV coordination and the rest of the two algorithms are for coordination of EVs within the day ${ }^{(7)}$.

Coordination of Plugged in EVs (PEV) charging and discharging is presented in ${ }^{(8)}$ to deal with the uncertainty in renewable energy generation and to improve power quality of the grid. $\mathrm{In}^{(9)}$, coordination of EVs and storage devices controlled by an electric distribution system is achieved by using mixed integer linear programming formulation. An improved optimal coordination method for large scale of PEV charging and discharging is proposed in ${ }^{(10)}$. A real-time charging station for EV charging and discharging is presented in ${ }^{(11)}$. A two-stage control scheme for PEV discharging is proposed in ${ }^{(12)}$ for peak load shaving. 
A decentralized optimal scheduling problem for PEV charging/discharging is solved by using economic model predictive control technique in ${ }^{(13)}$. Schedule for EV charging/discharging using particle swarm optimization is presented in ${ }^{(14)}$. A scheduling strategy for EV charging using moving window technique is proposed in ${ }^{(15)}$ considering forecasted load and price. An optimization model for coordination of EVs and renewable energy sources is proposed in ${ }^{(16)}$ this uses charging and discharging of EVs to reduce uneven patterns in equivalent load and increases renewable energy consumption.

The major limitation is lying in the scheduling problem of EVs are basically because of V2G operation which requires a primitive control of the charging/discharging behaviors of EVs to tackle the following issues- 1) Stochastic and variable regulation demand 2) Potential conflicts between EVs charging and suitable provision of the regulation service 3) Computational complexity and security issues incurred by the scheduling process of EVs. In this work, an optimal charging/discharging scheduling scheme is proposed to resolve all of these key issues and also to reshape the load profile to improve the overall regulation performance integrated with grid.

The relevant contributions and objectives of this work are-

1. To propose and evaluate the performance of centralized scheduling scheme for charging/discharging of EVs.

2. To propose and evaluate the performance of decentralized scheduling scheme for charging/discharging of EVs.

3. To bring the optimal coordination of EVs charging/discharging to reshape the load profile.

\section{Proposed Centralized Scheduling Scheme}

The main objective of the proposed centralized scheduling approach is to break the required charging time for 24 hours into several small charging intervals within the parking time, (Rezaei, 2014). In each scheduling slot, $\Delta t$, if enabled, a CS can charge the EV connected to it only for the duration of the scheduling slot providing a charging packet ${ }^{(17)}$. During each $\Delta t$, the algorithm assigns the starting time of each charging packet for all EVs in the parking areas and minimizing the peak demand along with the charging cost at the same time. Each EVs are charged according to a first come first serve service policy. The proposed scheduling technique is mathematically formulated below in section II-B. Centralized scheduling scheme is based on assumptions of perfect information regarding a known load and EVs at each interval.

\section{A. Scheme specifications}

In this scheme, one day scheduling of EV for charging and discharging is considered with a total of 24 hours with 24 intervals. The interval set is denoted by $\mathrm{L}$. Whereas, the length of each interval is taken for one hour which is denoted by 1 such that $l=1$ hour. On the other hand, V2G capable EV group can performs both charging and discharging in one day scheduling is denoted by $\mathrm{E}$. At the interval $\mathrm{n}$, the status of $\mathrm{EV}$ is also represented by $Y_{e n}(\forall e \in E, \forall n \in L)$. Where, $Y_{e n}$ is the charging/discharging power of EV e in the interval n. $P^{n}$ is the past interval set of n. If $Y_{e n} \geq 0$, then the status of $\mathrm{EV}$ at interval $\mathrm{n}$ is to charge the battery. If $Y_{e n}<0$, then the status of $\mathrm{EV}$ at interval $\mathrm{n}$ is to discharge the battery.

The plug in time of EV e into the charging spot is represented by $A_{e}^{p-i n}$. The plug out time of EV e into the charging spot is also denoted by $A_{e}^{p-o u t} . A_{e}$ is the set of total charging/discharging intervals of EV set that are on the schedule for 24 hours is represented by $A_{e}=A_{e}^{p-i n}+A_{e}^{p-\text { out }}$. Whereas, the battery initial energy level at the time of plug in $A_{e}^{p-i n}$ is denoted by $B_{e}^{f i n}$ should not exceed the capacity of the battery $B_{e}^{c a p}$. Energy ratio of EV e is given by $R_{e}=B_{e}^{f m} / B_{e}^{c a p}$ where $0 \leq R_{e} \leq 1$ . Energy ratio of EV e is always lies between 0 to $1 . A_{e}^{p-i n} B_{e}^{f i n}$ and $B_{e}^{c a p}$ are detected by charging station. $A_{e}^{p-o u t}$ and $R_{e}$ are provided by the customer to the charging station before charging is started. Then, the charging station identifies $A_{e}$ from $A_{e}^{p-i n}$, $A_{e}^{p-o u t}$ constraints. To determine whether EV is charging or discharging, a charging/discharging interval matrix is considered. $M \subset\{0,1\}^{|E|^{*} L \mid}$ where represents EV set and L represent interval set ${ }^{(16,18)}$.

$$
M_{\mathrm{en}}=\left\{\begin{array}{c}
1, \text { if } \mathrm{n} \text { is in charging region of } \mathrm{A}_{\mathrm{e}} \\
0, \text { if } \mathrm{n} \text { is in discharging region of } \mathrm{A}_{\mathrm{e}}
\end{array}\right.
$$

\section{B. Formulation of Centralized Scheme}

For centralized scheduling scheme of EV charging and discharging, following assumptions are considered based on reference $(16,18,19)$ 1) Agreement for V2G service between EV owners and the charging station 2) Plug in time and plug out time of all EVs in the EV set are known 3) Battery centralized scheduling for EV charging/discharging. 


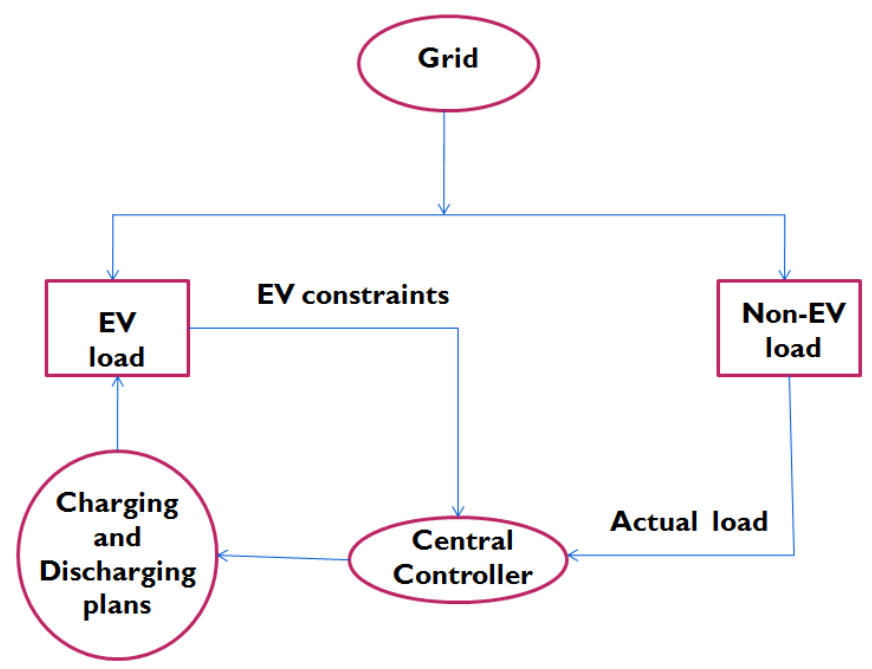

Fig 2. Illustration of the centralized scheduling scheme

The proposed centralized scheme is aimed to flatten the peaks and valleys of load profile by charging EV during light loads and discharging EV during peak loads to optimize charging/discharging power $Y_{e n}$, so as to minimize total load $X_{n}$ in the interval $\mathrm{n}$. The overall objective functions for the above cases are represented by-

$$
\begin{gathered}
\text { Total load; } X_{n}=L_{n}^{a}+\sum_{e \in E} Y_{e n} M_{e n},(\forall n \text { ò } \mathrm{L}, \forall e \in E) \\
\text { Instant energy; } 0 \leq B_{e}^{i n i}+\sum_{g=P^{n}} l Y_{e g} M_{e g} \leq B_{e}^{c a p},(\forall n \text { ò L }, \forall e \in E) \\
\text { Final energy; } B_{e}^{j i i}+\sum_{n=L} l Y_{e n} M_{e n} \geq R_{e} B_{e}^{c a p},(\forall n \text { ò L }, \forall e \in E) \\
-C^{\max } \leq Y_{e n} \leq C^{\max },(\forall \mathrm{n} \text { ò L }, \forall \mathrm{e} \in \mathrm{E})
\end{gathered}
$$

The four equations stated above represent the constraints for centralized optimal EV scheduling. Constraints in eq. (1) represents the total load included with EV load and non-EV load. Constraints in eq. (2) represents instant energy level of the EV battery which must not be zero or not greater than EV battery capacity $B_{e}^{\text {cap }}$. Constraints in eq. (3) represents the final energy level of EV battery which is not less than $R_{e} B_{e}^{c a p}$. Constraints in eq. (4) represents the lower bound $-C^{\max }$ and the upper bound $C^{\max }$ of the $Y_{e n}$. These constraints provide the centralized scheduling for EV charging/discharging for the day.

\section{Proposed Decentralized Scheduling Scheme}

Decentralized scheduling scheme is formulated optimally to schedule electric vehicle (EV) charging. The proposed scheme exploits the elasticity of EV loads to fill the peak in electric load profiles. So here we first formulated EV charging scheduling problem as an optimal control problem, whose objective is to impose a generalized notion of peak filling, and study properties of optimal charging profiles. Then we proposed a decentralized scheme to iteratively solve the optimal control problem. In each iteration, EVs are updated with their charging profiles according to the control signal forecasted by the utility. The scheme converges to optimal charging profiles irrespective of the specification of EVs. In centralized scheduling scheme, optimal scheduling is completely based on assumptions. First, the future arrival of EVs at any interval $\mathrm{n}$ in the day is unknown. Second, the future load at any interval $\mathrm{n}$ in the day is unknown. Third, it cannot handle a large number of EVs. So this scheme is not a practical solution for EV scheduling. For this reason, a decentralized scheme is also developed which overcomes all the abovestated setbacks. This scheme obtained the performance which is almost equal to the centralized scheme. Comparatively this scheme can be chosen as the practical scheduling scheme.

\section{A. Scheme Specifications}

Decentralized scheduling is based on the groups. According to the charging locations of EV fleet, they are divided into groups like a parking lot, residential garage. In this work, a group of $100 \mathrm{EVs}$ is chosen for analyzing the proposed scheme. Each group 
is headed by the local controller. All local controllers of each group are controlled by a central controller. Local controllers (LC) communicate with a central controller in the utility company. Using similar day analysis method central controller forecasts the load for the scheduling day and these forecasted load data is sent to all local controllers at the beginning of the day that comes under the central controller and also collects actual charging load for each EV.

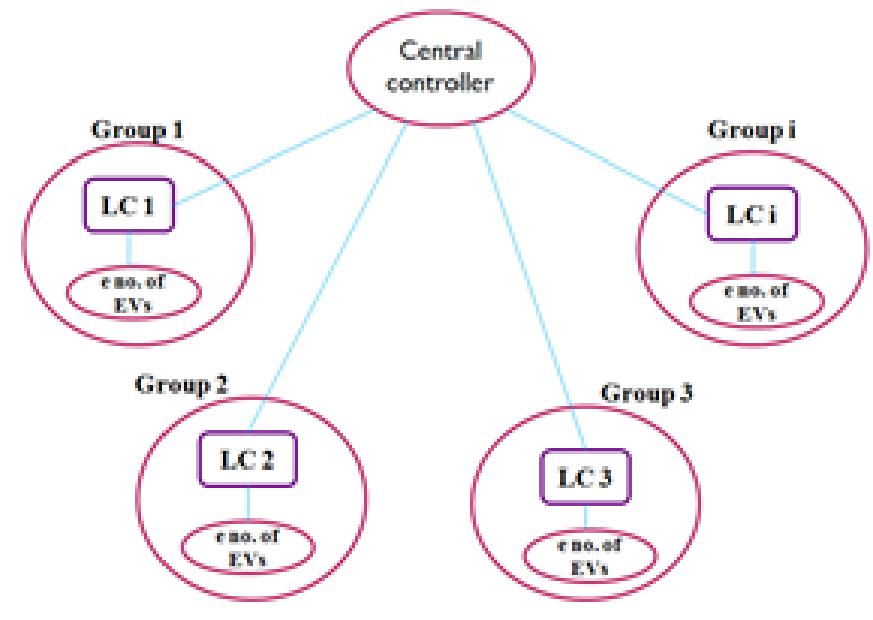

Fig 3. Illustration of the decentralized scheduling scheme

Each local controller contacts with other local controllers at the beginning of each interval $\mathrm{n}$ to collect EV information, current on going EV set $D_{i}^{(n)}$, current sliding window $S_{i}^{(n)}$ and current charging/discharging matrix $M_{e o}^{n}$ and then performs scheduling for EV charging/discharging. Local controller at the end of each interval reports actual charging load of each EV to the central controller. Let, there be i number of groups denoted as N. As every group performs independent scheduling with same objectives, one among them is chosen for evaluating decentralized scheduling. As like in the centralized scheme, future arrival of EVs are not known by the local controller in this scheme. So sliding window technique is used to determine charging powers for EVs at the beginning of every interval.

\section{B. Formulation of Decentralized Scheme}

At the starting of the interval, $n(\forall n \in L)$ two things are required to determine. One is the current on-going EV set $D_{i}^{(n)}$ and another is a current sliding window $S_{i}^{(n)}$. Each EV has certain charging/discharging period. The starting and finishing time of this period is denoted by $S_{e}^{D}, F_{e}^{D}$ and current time such that at the beginning of the interval $\mathrm{n}$ is denoted by $T^{\text {cur }}$. EVs which satisfies the conditions $S_{e}^{D} \leq T^{c u r}$ and $F_{e}^{D} \leq T^{c u r}$ are said to be in current on going EV set $D_{i}^{(n)}$. The current sliding window $S_{i}^{(n)}$ at the beginning of the interval $\mathrm{n}$ is the set of successive intervals between the starting time $S_{e}^{S}$ and finishing time $F_{e}^{S}$ of the sliding window. The starting time of the sliding window is $S_{e}^{S}=T^{c u r}$ and finishing time is $F_{e}^{S}=\max \left\{F_{e}^{D} \mid e \in D_{i}^{(n)}\right.$.

Figure 4 shows the on going EV set and sliding window at the beginning of interval 2. From Figure 4, it is concluded that EV-1 has completed the charging and the current on going EV set $D_{i}^{(2)}=\{E V s: 2,3,4\}$. Current sliding window $S_{i}^{(2)}=\{$ intervals $: 2,3,4,5\}$. To determine whether EV is charging or discharging, a charging/discharging interval matrix is considered which is represented by $M^{(n)} \subset\{0,1\}^{(n) *} S_{i}^{(n)}$

$$
M_{e o}^{n}=\left\{\begin{array}{l}
1, \text { if interval o is within } S_{i}^{(n)} \& \text { and charging period of } \mathrm{EV} \\
0, \text { otherwise }
\end{array}\right.
$$

Load at each interval is the forecasted load, obtained from averaging load values of similar weather conditions. The forecasted load is denoted as $L_{o}^{f}$ which helps to determine charging power in the current sliding window. The decentralized scheme is also aimed to flatten the peaks and valleys of load profile by charging EV during light loads and discharging during peak loads to optimize charging/discharging power $Y_{e o}$ so as to minimize total load $X_{e o}$ in the interval zero. Optimization for the $i^{\text {th }}$ group in 


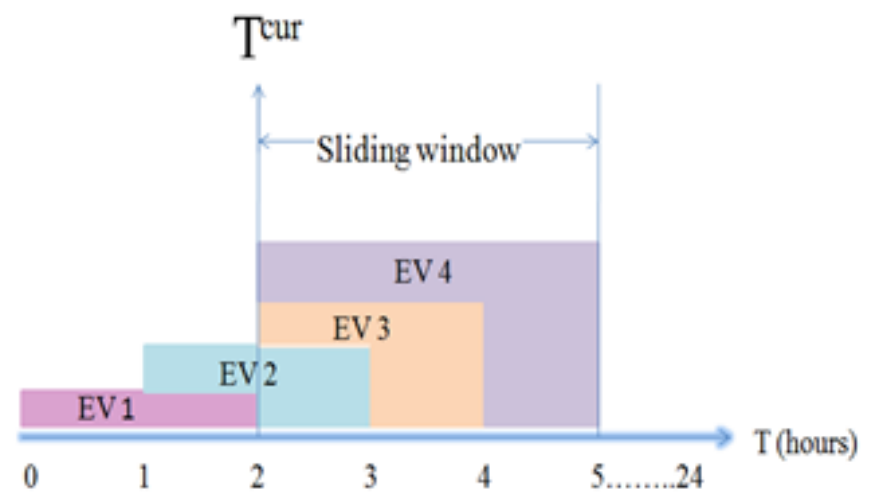

Fig 4. Representation of current ongoing EV set and current sliding window

$\mathrm{N}$ set, in terms of stated objectives, is given by-

$$
\begin{gathered}
\text { Total load: } X_{o}=L_{o}^{f}+\sum_{e \in D_{i}^{(n)}} Y_{e o} M_{e o}^{(n)}, o \in S_{i}^{(n)} \\
\text { Instant energy: } 0 \leq B_{e}^{(n) i n i}+\sum_{g \in P_{i}^{n}} l Y_{e g} M_{e g}^{(n)} \leq B_{e}^{c a p}, e \in D_{i}^{(n)}, 0 \in S_{i}^{(n)} \\
\text { Final energy: } B_{e}^{(n) i n i}+\sum_{\left.o \in S_{i}^{n}\right)} l Y_{e o} M_{e o}^{(n)} \geq R_{e} B_{e}^{c a p}, \forall e \in D_{i}^{(n)} \\
-C^{\max } \leq Y_{e o} \leq C^{\max }, \forall e \in D_{i}^{(n)}, 0 \in S_{i}^{(n)}
\end{gathered}
$$

The four equations stated above represent the constraints for decentralized optimal EV scheduling. Constraints in eq. (5) represents the total load included with EV load at the current sliding window and non EV load at the current interval. Constraints in eq. (6) represents the instant energy level of the EV battery which must not be equal to zero or not greater than EV battery capacity $B_{e}^{\text {cap }}$. Constraints in eq. (7) represents the final energy level of EV battery which is not less than $R_{e} B_{e}^{c a t}$. Constraints in eq. (8) represents the lower bound $-C^{\max }$ and the upper bound $C^{\max }$ of the $Y_{e o}$. These constraints provide the decentralized scheduling for EV charging/discharging for the day.

The merits of this scheme can be handled with a large number of EV population and at each interval local controller updates charging power by gathering $\mathrm{EV}$ information. Thus, this scheme responds quickly to the dynamic EV arrivals.

\section{Result and Discussion}

The following settings are considered for the purpose of evaluating the proposed two schemes for EV charging/discharging. Scheduling is carried out for a day from 1:00 am midnight, by dividing 24 hours into 24 intervals. Each interval lasts for one hour. The load data is taken from microgrid in Toronto by a factor of $1 / 500$ which is similar to ${ }^{(17)}$. All EVs in two schemes belongs to company Nissan leaf 2017 model with battery capacity of $30 \mathrm{kWh}$ and 107 miles range ${ }^{(19)}$. Same specifications are considered for all EVs. EVs from company Nissan leaf is chosen because they have zero percentage of carbon emissions. Expected battery energy at the end of the charging period is 90 percentage of the battery capacity. Maximum charging power for each EV is set to $12 \mathrm{~kW}$ and also $C^{\max }=12 \mathrm{~kW}$.

Table 1. Arrival and departure time of first five EVs

\begin{tabular}{llll}
\hline S. N & EV No. & $\begin{array}{l}\text { EV Arrival time } \\
\text { (Specific Timing of Arrival in 24 Hours) }\end{array}$ & $\begin{array}{l}\text { EV Departure Time } \\
\text { (Specific Timing of Departure in 24 Hours) }\end{array}$ \\
\hline 1 & 1 & 2 & 14 \\
2 & 2 & 2 & 14 \\
3 & 3 & 11 & 23 \\
4 & 4 & 13 & 24 \\
5 & 5 & 18 & 24 \\
\hline
\end{tabular}


Table 2. EV specifications

\begin{tabular}{lllll}
\hline EV model & No. of EVs & Battery Capacity & Range & Maximum Charging Power \\
\hline Nissan leaf 2017 & 200 & 30kWh & 107 miles & $12 \mathrm{~kW}$ \\
\hline
\end{tabular}

Modeling of EV information such as arrival time, charging period and the initial energy is as follows. Though EV arrival is evenly distributed among all intervals, expected chances for the arrival of EVs is less than 30 number of EVs for each interval. Each EV charging period lies in between 4 to 12 hours and the initial energy of each EV lies in between 0 to $80 \%$ of the battery capacity. Number of EVs in EV fleet is set to 200.

Centralized scheduling scheme requires perfect information of EV and load so that the actual load is used in simulation. However, actual load in the future interval is impossible to determine. In decentralized scheduling scheme, forecasted loads are used in the simulation. Hence, this scheme is called practical solution of EV scheduling. Figure 5 shows the comparison of actual and forecasted loads. The actual load data is taken from microgrid in Toronto by a factor of $1 / 700^{(17)}$. Using similar day analysis method central controller forecasts the load by averaging the 8 days load. The mean relative error between the actual loads and forecasted loads is $e=(1 /|L|) \sum_{n \in L}\left|L_{n}^{f}-L_{n}^{a}\right| / L_{n}^{a} 0.0234$, which is quite small and better prediction.

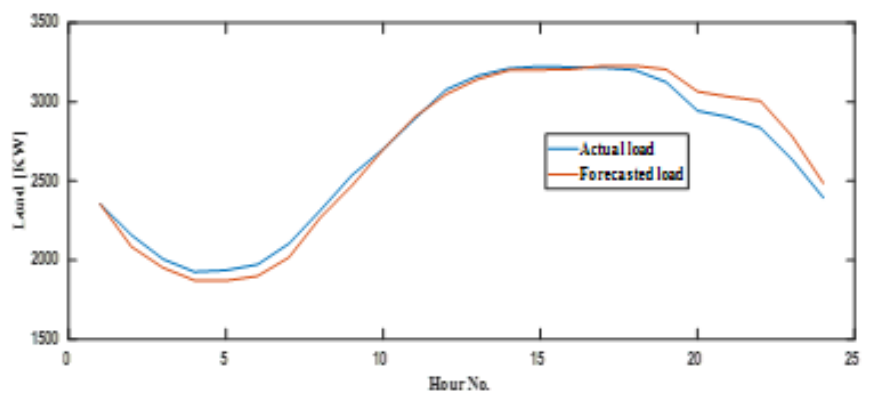

Fig 5. Comparison of actual load and forecasted load

Table 3. EVload, Total load at each interval in centralized scheme and decentralized scheme (from simulation)

\begin{tabular}{|c|c|c|c|c|}
\hline \multirow[t]{2}{*}{ Interval } & \multicolumn{2}{|l|}{ EV load $(\mathrm{kW})$} & \multicolumn{2}{|l|}{ Total load (kW) } \\
\hline & Centralized Scheme & Decentralized Scheme & Centralized Scheme & Decentralized Scheme \\
\hline 1 & -78.13363108409 & -122.735931457548 & 2276.41092605877 & 2231.80862568531 \\
\hline 2 & 119.103093079389 & -62.5192363242416 & 2276.41012165082 & 2094.78779224719 \\
\hline 3 & 269.837650180049 & 141.022085683165 & 2276.40980732291 & 2147.59424282602 \\
\hline 4 & 350.494527418265 & 327.700753287288 & 2276.40969884684 & 2253.61592471586 \\
\hline 5 & 340.275007370227 & 430.038921325179 & 2276.41005022737 & 2366.17396418232 \\
\hline 6 & 304.361456242981 & 450.206838442673 & 2276.41061338584 & 2422.25599558553 \\
\hline 7 & 204.581941383818 & 346.806650021991 & 2308.00869852668 & 2450.23340716485 \\
\hline 8 & 270.971344672741 & 270.971373913276 & 2585.05688752988 & 2585.05691677042 \\
\hline 9 & 238.478880154260 & 238.479063817813 & 2773.80275158283 & 2773.80293524638 \\
\hline 10 & 161.466231187618 & 161.466054210181 & 2863.95064547333 & 2863.95046849590 \\
\hline 11 & 167.591450766293 & 167.591493418123 & 3059.86196505201 & 3059.86200770384 \\
\hline 12 & 91.5884753476354 & 192.887098696143 & 3168.74083249049 & 3270.03945583900 \\
\hline 13 & 4.50044462949983 & 79.7046293132441 & 3168.74250177236 & 3243.94668645610 \\
\hline 14 & -43.183105714434 & -38.1785872272166 & 3168.74303714271 & 3173.74755562993 \\
\hline 15 & -56.176903299617 & -43.0880192799744 & 3168.74316812895 & 3181.83205214860 \\
\hline 16 & -51.111912604768 & -62.5652469708220 & 3168.74320168095 & 3157.28986731489 \\
\hline 17 & -47.107606516604 & -109.275903966870 & 3168.74313634054 & 3106.57483889027 \\
\hline 18 & -32.530073162933 & -123.901871047992 & 3168.74294112278 & 3077.37114323772 \\
\hline 19 & 43.5165682103781 & -89.3744463320328 & 3168.74243963895 & 3035.85142509654 \\
\hline 20 & 225.233676171407 & 196.199860687819 & 3168.74160474284 & 3139.70778925925 \\
\hline 21 & 265.123823151481 & 263.070589196822 & 3168.74089458005 & 3166.68766062539 \\
\hline 22 & 333.576812585843 & 314.585238968978 & 3168.74034115727 & 3149.74876754041 \\
\hline 23 & 531.247146319109 & 684.613859633050 & 3168.73966060482 & 3322.10637391876 \\
\hline
\end{tabular}

Continued on next page 
Table 3 continued

\begin{tabular}{lllll}
\hline 24 & 575.999882539769 & 575.999906264426 & 2970.85009682548 & 2970.85012055014 \\
\hline
\end{tabular}

EVs charging load in centralized scheduling scheme, at each interval is shown in Figures 6 and 7 show EVs charging load in the decentralized scheme, at each interval. Figure 8 shows the total load in centralized scheduling scheme i.e, a combination of both EV and Non EV load. Figure 9 shows the total load in decentralized scheduling scheme. As both schemes tend to charge the battery during light load and discharge during peak loads, total load curve is reshaped, and this is clearly observed by comparing total load curve with actual load curve. Both centralized and decentralized schemes reshape load curve at 1-7 and 12-23. The peak comparison: base load $=3224.92 \mathrm{~kW}$, in centralized scheme peak total load, is $3168.74 \mathrm{~kW}$ and in decentralized scheme peak, the total load is $3367.74 \mathrm{~kW}$.

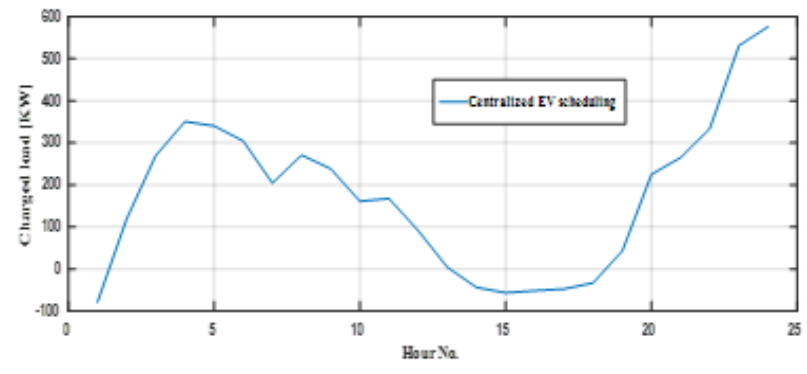

Fig 6. Total power output of 200 EVs in centralized scheme

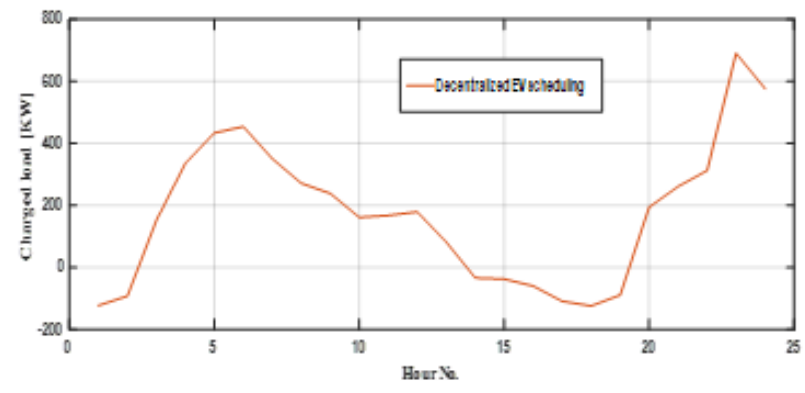

Fig 7. Total power output of $200 \mathrm{EVs}$ in a decentralized scheme

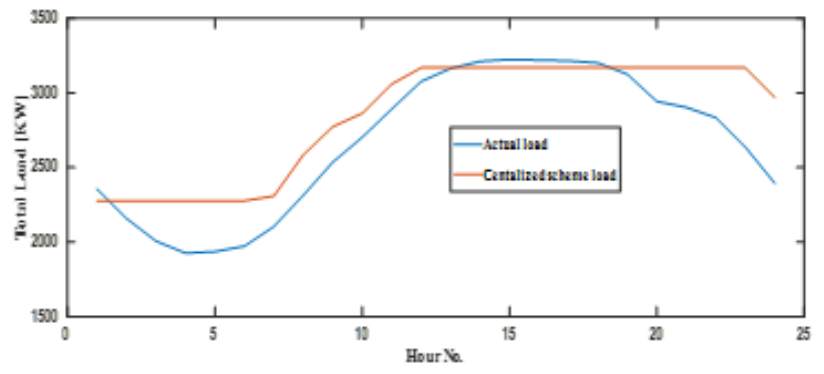

Fig 8. Comparison of total load (EV and Non-EV) in a centralized scheme with actual load

Proposed centralized scheme achieves better results than decentralized scheme as shown in Figure 10 . However, decentralized scheme results are nearly equal to centralized scheme results, which is the practical solution for EV charging/discharging scheduling. Charging power of randomly chosen EV (eg., EV 65) in the centralized scheme is shown in Figure 11. Charging power of randomly chosen EV (eg., EV 65) in the decentralized scheme is shown in Figure 12. Whereas, Figures 13 and 14 show that the energy variation of randomly chosen EV (eg., 65) in the centralized scheme and decentralized scheme. 


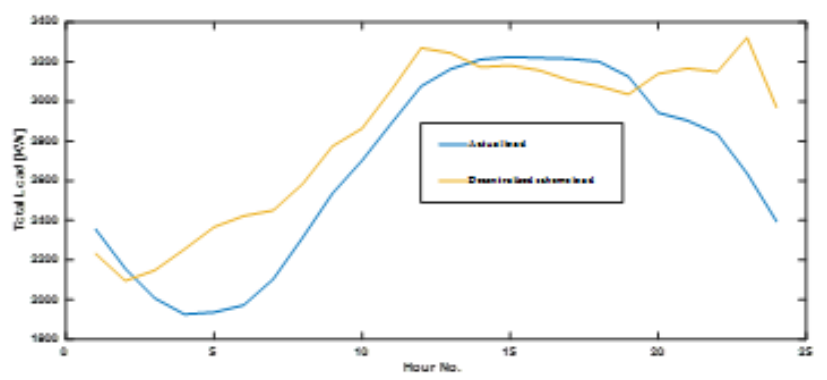

Fig 9. Comparison of total load (EV and Non-EV) in a decentralized scheme with actual load

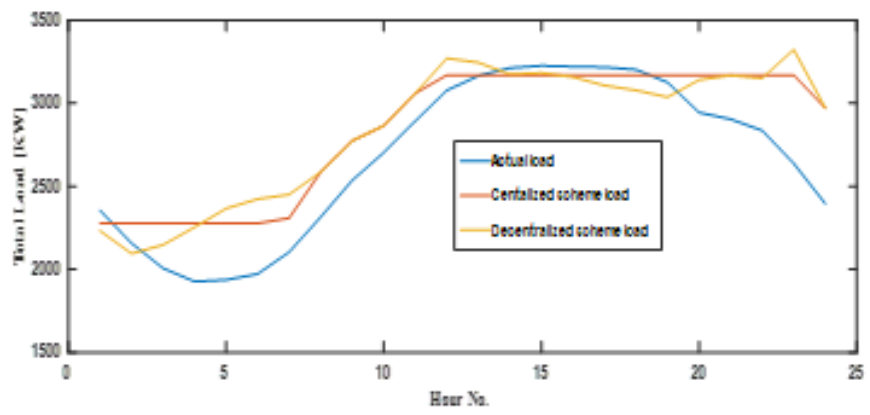

Fig 10. Total load comparison of centralized and decentralized schemes

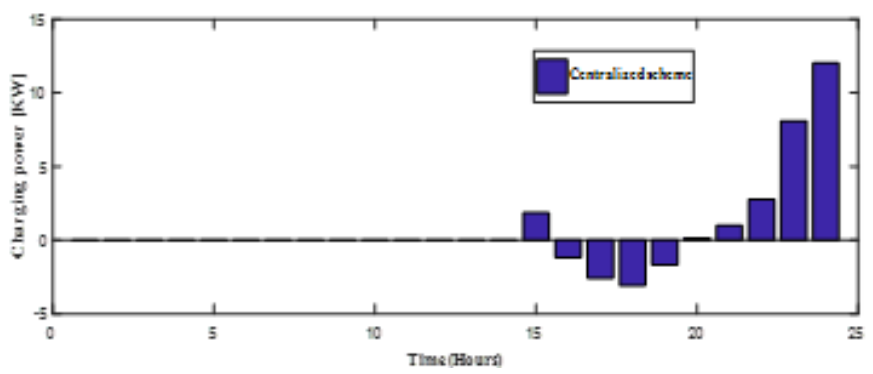

Fig 11. Rate of Charging/Discharging of EV- 65 in centralized scheme

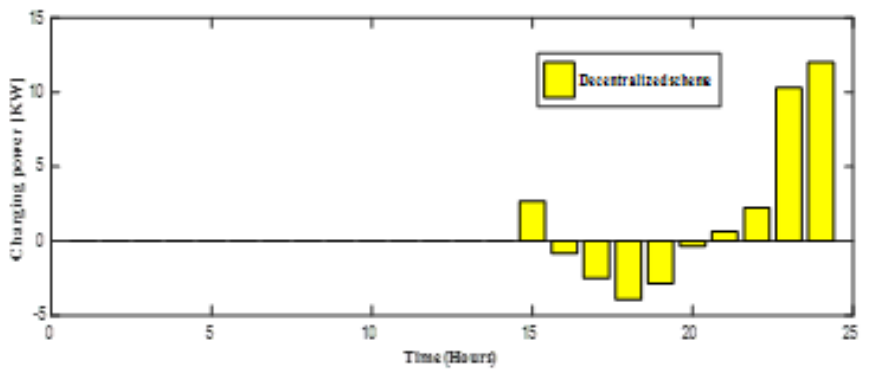

Fig 12. Rate of Charging/Discharging of EV- 65 in a decentralized scheme 


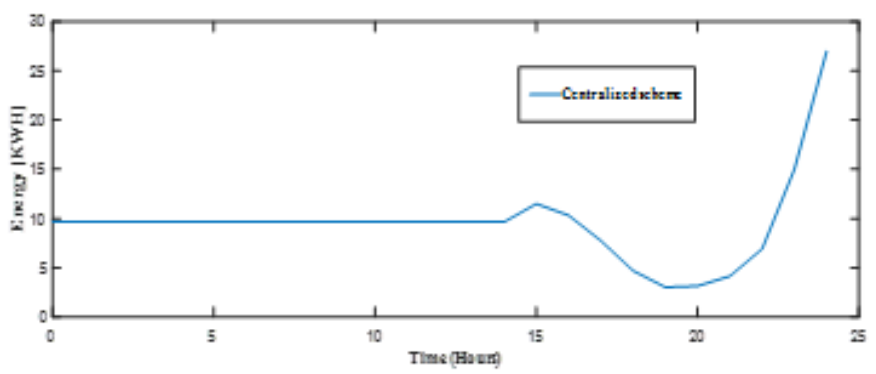

Fig 13. Energy levels of EV-65 in a centralized scheme

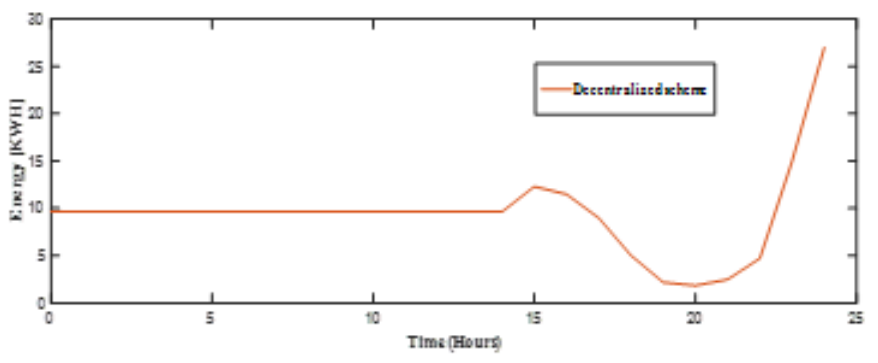

Fig 14. Energy levels of EV-65 in a decentralized scheme

\section{Conclusion}

Optimal scheduling schemes require perfect information of EV and load so that the actual load can be used in simulation. However, actual load in the future interval is impossible to determine. In decentralized scheduling scheme, forecasted loads are used in the simulation. Hence, this scheme is called practical solution of EV scheduling. Two scheduling methods such as centralized and decentralized schemes are proposed in this study. Proposed schemes are evaluated in Matlab simulations for one-day scheduling of EV charging and discharging. From simulation results, it is concluded that both schemes are achieving good results in terms of EV scheduling and load reshaping but the centralized scheme is accurate in results. Whereas, decentralized scheme results are nearly equal to the centralized scheme.The centralized scheme requires perfect information regarding EV and load which is not possible in practice. Overall, the decentralized scheme is based on forecasted data which can give the practical solution.

\section{References}

1) Global Climate Change: vital signs of the planet. . Available from: https://climate.nasa.gov.

2) Nguyen HNT, Zhang C, Mahmud MA. Apel Mahmud Optimal Coordination of G2V and V2G to Support Power Grids with High Penetration of Renewable Energy. IEEE Transactions on Transportation Electrification. 2015;1(2):188-195. Available from: 10.1109/TTE.2015.2430288.

3) Said D, Mouftah HT. A Novel Electric Vehicles Charging/Discharging Scheme with Load Management Protocol. In: and others, editor. Proceedings of IEEE International Conference on Communication (ICC). 2017;p. 1-6. doi:10.1109/ICC.2017.7997117.

4) Zhang W, Quan H, Gandhi O, Gallegos CDR, Srinivasan D, Weng Y. Dynamic and Fast Electric Vehicle Charging Coordinating Scheme Considering V2G Based Var Compensation. IEEE Conference on Energy Internet and Energy System Integration. 2017;(EI2):26-28. Available from: 10.1109/EI2.2017.8245686.

5) Jozi F, Mazlumi K, Hosseini H. Charging and discharging coordination of electric vehicles in a parking lot considering the limitation of power exchange with the distribution system. In: and others, editor. IEEE 4th International Conference on Knowledge-Based Engineering and Innovation (KBEI). 2017;p. 937-941.

6) Wang L, Sharkh S, Chipperfield A. A*-Based Optimal Coordination of Vehicle-to-Grid Batteries and Renewable Generators in a Distribution Network. IEEE 26th International Symposium on Industrial Electronics (ISIE). 2017;p. 43-50. Available from: 10.1109/ISIE.2017.8001221.

7) Karfopoulos EL, Panourgias KA, Hatziargyriou ND. Distributed Coordination of Electric Vehicles providing V2G Regulation Services. IEEE Transactions on Power Systems. 2016;31(4):2834-2846. Available from: https://dx.doi.org/10.1109/tpwrs.2015.2472957.

8) Li H, Zheng C, Zhang X, Chen X, Chang X. Research on Multi-Objective Optimization Coordination of Plug-in Hybrid electric Vehicle and Distributed generation. In: Proceedings of 5th international conference on Electric Utility Deregulation and Restructuring and Power Technologies (DRPT). 2015;p. 2608-2613. doi:10.1109/DRPT.2015.7432688.

9) Sabillón CF, John A, Franco F, Rider MJ, Romero R. A MILP Model for Optimal Charging Coordination of Storage Devices and Electric Vehicles Considering V2G Technology. In: Proceedings of IEEE 15th International Conference on Environment and Electrical Engineering (EEEIC). 2015;p. 60-65. doi:10.1109/EEEIC.2015.7165336. 
10) Chen Z, Hu Z. Optimal Coordination of Charging and Discharging Control of Large-scale Plug-in Electric Vehicles. In: IEEE Conference and Expo Transportation Electrification Asia-Pacific (ITEC Asia-Pacific). 2014;p. 1-6. doi:10.1109/ITEC-AP.2014.6940670.

11) Singh M, Kumar P, Kar I, Kumar N. A Real-time Smart Charging Station for EVs Designed for V2G Scenario and its Coordination with Renewable Energy Sources. In: Proceedings of IEEE Power and Energy Society General Meeting (PESGM. 2016;p. 17-21. doi:10.1109/PESGM.2016.7741479.

12) Erdogan N, Erden F, Kisacikoglu M. A fast and efficient coordinated vehicle-to-grid discharging control scheme for peak shaving in power distribution system. Journal of Modern Power Systems and Clean Energy. 2018;6(3):555-566. Available from: https://dx.doi.org/10.1007/s40565-017-0375-z.

13) Sha’aban YA, Ikpehai A, Adebisi B, Rabie KM. Bi-Directional Coordination of Plug-In Electric Vehicles with Economic Model Predictive Control. Energies. 2017;10(10). Available from: https://doi.org/10.3390/en10101507.

14) Liu D, Wang Y, Shen Y. Electric Vehicle Charging and Discharging Coordination on Distribution Network Using Multi-Objective Particle Swarm Optimization and Fuzzy Decision Making. Energies. 2016;9(3). Available from: https://doi.org/10.3390/en9030186.

15) Ma C, Rautiainen J, Dahlhaus D, Lakshman A, Toebermann JC, Braun M. Online Optimal Charging Strategy for Electric Vehicles. Energy Procedia. 2015;73:173-181. Available from: https://dx.doi.org/10.1016/j.egypro.2015.07.667.

16) Jiang X, Wang J, Han Y, Zhao Q. Coordination Dispatch of Electric Vehicles Charging/Discharging and Renewable Energy Resources Power in Microgrid. Procedia Computer Science. 2017;107(2):157-163. Available from: https://dx.doi.org/10.1016/j.procs.2017.03.072.

17) Website for Toronto Hydro-Electric System. . Available from: http://www.torontohydro.com/.

18) He Y, Venkatesh B, Guan L. Optimal Scheduling for Charging and Discharging of Electric Vehicles. IEEE Transactions on Smart Grid. 2012;3(3):1095-1105. Available from: https://dx.doi.org/10.1109/tsg.2011.2173507.

19) 2017 Nissan Leaf specs . 2017. Available from: https://www.greencarreports.com/news/1107264_2017-nissan-leaf-specs-all-30-kwh-batteriesotherwise- unchanged. 